\title{
Seis tesis no convencionales sobre participación
}

\section{Kliksberg, Bernardo*}

\section{Resumen}

La participación ha sido reconocida en el escenario mundial como estrategia maestra de la gerencia de excelencia, incluso por organismos internacionales como el Banco Mundial, Banco Interamericano de Desarrollo, y la organización para la Cooperación y el Desarrollo. Al respecto en este trabajo se discuten las siguientes tesis: La participación de los involucrados (comunidad y trabajadores), da resultados muy superiores a otros modelos organizacionales; tiene ventajas comparativas que derivan de bases muy precisas y facilita no sólo el logro de metas concretas sino de suprametas; la participación es un núcleo central de la gerencia del año 2000, implicando un cambio de paradigma; sin embargo existen contradicciones entre el discurso y la realidad; la participación por esto enfrenta fuertes resistencias e intereses; se requieren políticas y estrategias orgánicas activas para hacerla avanzar, no sólo por las ventajas que ella representa, sino porque la participación es intrínseca a la naturaleza humana, implica devolver a la población un derecho que le pertenece.

Palabras clave: Participación, gerencia, modelo organizacional, eficiencia.

Recibido: $98-09-07$ Aceptado: 98-10-14

* Profesor Honorario de la Universidad Nacional de Buenos Aires, Doctor Honoris Causa de la Universidad Nacional Experimental Rafael María Baralt (UNERMB), Profesor Emérito de la Universidad del Congreso, Director del Proyecto Regional de las Naciones Unidas para América Latina de "Modernización del Estado y Gerencia Social" (PNUD-CLAD). Actualmente es Coordinador General del Instituto interamericano de Desarrollo Social (BID). 


\section{Six Non-Conventional Thesis as to Participation}

\section{Abstract}

Participation has been recognized world-wide as a master strategy in the excellence management, even by the World Bank, the Interamerican Development Bank, and the organization for Cooperation and Development. In this paper the following thesis are discussed: Participation of those involved (community and workers) gives far superior results compared to other organizational models; It has comparative advantages which derive from very specific bases and which facilitate not only the completion of very specific goals, but also supra-goals; Participation is a central nucleus of management in the year 2000 , implying a change in the paradigm; however, there are contradictions between the discourse and the reality; for this reason participation confronts strong resisytence and powerful interests; active, organic policies and strategies are required to help its advance, not only because of the advantages it offers, but because participation is intrinsic to human nature, and implies returning to the people a right which belongs to them.

Key words: Participation, management, organizational model, efficiency.

\section{La participación en el centro del escenario}

Hasta hace pocos años la participación comunitaria en el desarrollo económico y social era un tema altamente polémico, objeto de fuertes controver sias, fácilmente susceptible de rápidos etiquetamientos ideológicos. Una de sus descalificaciones más frecuentes era considerarla integrante del reino de las "utopias" sin sentido de realidad. Actualmente se está transformando en un nuevo consenso. Gran parte de los organismos internacionales de mayor peso están adoptando la participación como estrategia de acción en sus declaraciones, proyectos, $\theta$ incluso en diversos casos están institucionalizándola como política oficial. Entre ellos, el Banco Mundial publicó en 1996 un libro "maestro" sobre participa- ción. Señala que presenta "la nueva dirección que el Banco está tomando en apoyo de la participación", y resalta que "la gente afectada por intervenciones para el desarrollo debe ser incluida en los procesos de decisión". Su Departamento de Políticas preparó estrategias y un Plan de Acción a largo plazo en donde se formulan lineamientos muy concretos. Entre ellos, que el Banco fortalecerá las iniciativas de los prestatarios que fomenten la incorporación de los métodos participativos en el desarrollo, que la participación de la comunidad será un aspecto explicito del diálogo con el pais y de las Estrategias de Ayuda al pais, y que el Banco fomentará y financiarà asistencia técnica que fortalezca el involucramiento de la gente de escasos recursos y otros afectados por el proyecto. Ya desde años anteriores el sistema de la Naciones Unidas 
había integrado la promoción de la participación como un eje de sus programas de cooperación técnica en el campo económico y social. Los informes sobre Desarrollo Humano que viene publicando desde 1990 y que examinan problemas sociales fundamentales del planeta, indican en todos los casos a la participación como una estrategia imprescindible en el abordaje de los mismos. El Banco Interamericano de Desarrollo editó en 1997 un Libro de Consulta sobre Participación. En la introducción se indica que "La participación no es simplemente una idea si no una nueva forma de cooperación para el desarrollo en la década del $90^{\prime \prime}$ : Se destaca el peso que se proyecta asignarle: "La participación en el desarrollo y su práctica reflejan una transformación en la manera de encarar el desarrollo a través de los programas y proyectos del Banco". La Organización para la Cooperación y el Desarrollo (OECD 1993) reconoce que la "participación más amplia de todas las personas es el principal factor para fortalecer la cooperación para el desarrollo". EIPrograma de las Naciones Unidas para el Desarrollo (PNUD 1993) destaca que: "La participación es un elemento esencial del desarrollo humano" y que la gente "desea avances permanentes hacia una participación total".

Otros organismos de cooperación internacional globales, regionales, subregionales y nacionales están sumándose al nuevo consenso. Pero el proceso no se limita a los dadores de cooperación y préstamos para el desarrollo. Va mucho más lejos. En las sociedades latinoamericanas se está dando un crecimiento continuo de abajo hacia arriba, de la presión por estructuras participatorias, y una exi- gencia en aumento sobre el grado de genuinidad de las mismas. La población demanda participar, $y$ entre otros aspectos una de las causas centrales de su interés y apoyo a los procesos de descentralización en curso, se halla en que entrevé que ellos pueden ampliar las posibilidades de participación si son adecuadamente ejecutados.

Como todos los cambios significativos en la realidad, esta relectura de la participación como una estrategia maestra de desarrollo tiene anciajes profundos en necesidades que surgen de la realidad. América Latina está llegando a fin del siglo $X X$ con un cuadro social extremadamente delicado. Como lo señalaron expresamente los Presidentes de todo el continente en la reciente Cumbre de Santiago (1998): "Superar la pobreza continúa siendo el mayor desafío que confronta nuestro hemisferio".

La referencia a la pobreza como el mayor problema abierto tiene bases en los hechos. Según el informe al respecto de una comisión especial presidida por Patricio Aylwin (1995), casi la mitad de los habitantes de la región están por debajo de la línea de pobreza, y el $41 \%$ padece de algún grado de desnutrición. Según UNICEF el $60 \%$ de los niños son pobres. La escolaridad promedio es de 5,2 años (menor a primaria completa). Según el Banco Mundial (1996) 2.200.000 niños nacen sin que sus madres cuenten al dar a luz con asistencia médica de ningún tipo, con los consiguientes impactos en las tasas de mortalidad materna e infantil. Asimismo, La región es caracterizada mundiaimente como la más desigual del mundo. Se observan severas iniquidades en la distribución de ingresos, el acceso a 
activos productivos, el crédito y las posibilidades de obtener una educación de calidad razonable ${ }^{1}$. Este panorama de pobreza e iniquidad pronunciada inadmisible en un sistema democrático como el que ha alcanzado la región después de largas luchas, y bloqueador del desarrollo, reclama respuestas urgentes e imaginativas y ha sido el motor fundamental del nuevo interés surgido en torno de la participación comunitaria. La experiencia muchas veces frustrada o de resultados limitados en las políticas y proyectos de enfrentamiento de la pobreza, ha dejado como uno de sus saldos favorables la constatación de que en la participación comunitaria puede haber potencialidades de gran consideración para obtener logros significativos y al mismo tiempo mejorar la equidad.

La participación siempre tuvo en América Latina una legitimidad de carácter moral. Desde amplios sectores se planteó con frecuencia como un derecho básico de todo ser humano, con apoyo en las cosmovisiones religiosas y éticas predominantes en la región. También tuvo continuamente una legitimidad política. Es una vía afín con la propuesta histórica libertaria de los padres de las naciones de la región, y con el apego consistente de la misma al ideal democrático. Ahora se agrega a dichas legitimidades otra de carácter diferente, que no excluye las ante- riores sino se suma a ellas. La participación tiene una legitimidad macroeconómica y gerencia. Es percibida como una alternativa con ventajas competitivas netas para producir resultados en relación a las vías tradicionalmente utilizadas en las políticas públicas. Ello pone la discusión sobre la participación en un encuadre diferente al de décadas anteriores. No se trata de una discusión entre utópicos, sino de poner al servicio de los severos problemas sociales que hoy agobian a buena parte de la población, los instrumentos más efectivos, y alli aparece la participación, no como "imposición de algún sector, sino como oportunidad".

Como toda "oportunidad", su movilización efectiva enfrenta fuertes resistencias de diversa índole. Su presencia es evidente observando la vasta brecha que separa en América Latina el "discurso" sobre la participación de las realidades de implementación concreta de la misma. En el discurso el consenso parece total, y la voluntad de llevarla adelante potente. En la realidad el discurso no ha sido acompañado por procesos serios y sistemáticos de implementación. Esa distancia tiene entre sus causas principales la presencia silenciosa de bloqueos considerables al avance de la participación.

Este trabajo procura aportar a la reflexión abierta que es imprescindible llevar a cabo en la región hoy para ayudar a

Entre muchas otras afirmaciones coincidentes al respecto, destaca Shadid Javed Burki, Vicepresidente para América Latina del Banco Mundial (1996): "La región de América Latina y el Caribe tiene la más pronunciada disparidad en los ingresos de todas las regiones en desarrollo en el mundo", y resalta editorialmente el New York Times (1997) que América Latina es la región "que tiene la mayor brecha entre ricos y pobres". 
que las promesas de la participación comunitaria puedan hacerse realidad en beneficio de los amplios sectores desiavorecidos de la región. Para ello plantea una serie de tesis sobre aspectos claves del tema. Tratar de poner a foco en qué consiste la nueva legitimidad de la participación, resaltar cómo forma parte de un mo. vimiento más general de replanteo de la misma en la gerencia de avanzada, identificar algunas de las principales resistencias subterráneas a la participación, y sugerir estrategias para escalarlas.

El objetivo de fondo no es exhaustivizar ninguno de los temas planteados, sino ayudar a construir una agenda de discusión históricamente actualizada sobre la materia, y estimular el análisis colectivo de la misma.

\section{Primera tesis: La participación da resultados}

Según enseña la experiencia concreta, promover y poner en marcha modelos participativos genuinos, significa en definitiva gerenciar con excelencia. La participación da resultados muy superiores en el campo social a otros modelos organizacionales de corte tradicional como los burocráticos y los paternalistas.

Uno de los estudios cercanos más significativos al respecto es llevado a cabo por el Banco Mundial sobre 121 proyectos de dotación de agua potable a zonas rurales en 49 paises de Asia, Africa y América Latina (1994, 1995). Los proyectos estaban apoyados por 18 agencias internacionales. Se seleccionó el agua como tema central de la evaluación, por cuanto la falta de acceso a agua potable es un problema que afecta a vastos sectores de población pobre, tiene el más alto rango de importancia y hay una larga historia de programas en esa área.

La investigación recogió data sistemática sobre dichos proyectos y realizó análisis cuantitativos y cualitativos comparativos entre ellos. Al mismo tiempo efectuó exámenes de la evolución de los proyectos durante períodos en algunos casos superiores a diez años. Se estudiaron 140 variables, y se introdujeron diversas precauciones metodológicas para evitar efectos "halo" $y$ otros posibles sesgos. Los resultados finales pueden apreciarse en el Cuadro 1.

Como se observa, el cuadro clasifica a los proyectos según el nivel de participación de los beneficiarios en proyectos de baja, mediana y alta participación. A su vez cruza esa clasificación con atra que es la identificación de los proyectos que tuvieron baja, mediana y alta efectividad en términos de las metas buscadas. En los proyectos con baja participación sólo el $3 \%$ tuvieron alta efectividad mientras en los proyectos con mediana participación el $31 \%$ tuvo alta efectividad, es decir, se multiplicó por $10 \mathrm{la}$ efectividad. En los proyectos con alta participación la efectividad llega a su tope, el $81 \%$ de los proyectos tuvieron alta efectividad. El grado de efectividad alcanzada multiplica por 27 al obtenido en los de baja participación, y por 2,6 al de los proyectos con mediana participación.

La participación de la comunidad cambió radicalmente los grados de logro de metas de los proyectos.

Según indica la investigación, algunos de sus resultados fueron: 


\section{Cuadro 1}

Efectividad según los Niveles de Participación de la Comunidad en Proyectos Rurales de Agua

\begin{tabular}{|c|c|c|c|c|c|}
\hline \multirow[t]{2}{*}{ Variable } & \multicolumn{5}{|c|}{ Grado de participación de los beneficios } \\
\hline & & Bajo & Mediano & Alto & $\begin{array}{l}\text { Total de } \\
\text { proyectos }\end{array}$ \\
\hline \multirow{3}{*}{$\begin{array}{l}\text { Grado de } \\
\text { efectividad de } \\
\text { los proyectos }\end{array}$} & Bajo & 21 & 6 & 0 & $27(22 \%)$ \\
\hline & Mediano & 15 & 34 & 5 & $54(45 \%)$ \\
\hline & Alto & 1 & 18 & 21 & $40(33 \%)$ \\
\hline \multicolumn{2}{|c|}{ Total de proyectos } & $37(31 \%)$ & $58(48 \%)$ & $26(21 \%)$ & $121(100 \%)$ \\
\hline
\end{tabular}

Fuente: Deepa Narayan. The Contriution of People's Participation: 121 Rural Water Supply Projects. World Bank, 1994.

- el mantenimiento de los sistemas de agua instalados en buenas condiciones (factor crucial en esta materia)

- la extensión del porcentaje de población alcanzada

- la mayor igualdad en el acceso

- beneficios económicos generales

- beneficios ambientales

Por otra parte, señalan los investigadores, la participación fue un factor fundamental de empoderamiento de la comunidad. Influyó fuertemente en:

- la adquisición por parte de los miembros de la comunidad de nuevas habilidades organizacionales y de destrezas relacionadas con el manejo del agua.

- el fortalecimiento de la organización comunitaria.

Los resultados indican que la participación no debe limitarse a algunas etapas del proyecto. La efectividad aumenta cuando está presente en todo el ciclo del proyecto. Por ello los serios problemas que encuentran los proyectos de agua que son diseñados sin consulta a los beneficiarios y en los que se espera des- pués que la comunidad no consultada se hará responsable por su operación y mantenimiento.

El cambio en la aplicación de la participación generó variaciones sustanciales a lo largo de la vida de los proyectos. Entre otros casos examinados, en su fase 1 el proyecto del Aguthi Bank en Kenya fue conducido $\sin$ la participación de la comunidad. Estuvo plagado de problemas, demoras en la construcción, sobrecostos, y desacuerdo sobre los métodos de pago de los consumidores, y tuvo que paralizarse. Fue rediseñado y los líderes locales se autoorganizarón en el Aguthi Water Committee. Trabajando con el equipo del proyecto movilizaron el apoyo de la comunidad, la cual comenzó a contribuir con trabajo y aportes económicos. Desarrollada de ese modo, la fase 2 del proyecto se completó en el tiempo y dentro del presupuesto fijados. La comunidad paga las tarifas mensuales acordadas por el servicio, y el mantenimiento del sistema y cogestiona ambos con el Gobierno. En Timor, Indonesia, el programa Wanita, Air Dan Sanitasi se propuso ayu- 
dar a grupos de la comunidad para que administraran su propio sistema de agua. Se formaron grupos pero los equipos gubernamentales demoraban en llegar. Los grupos incrementaron su participación y comenzaron a operar solos. Negociaron derechos de agua con un grupo vecino, consiguieron material de construcción, $y$ construyeron tanques de agua con una limitada asistencia técnica.

La opción por la participación en lugar de otras modalidades posibles se considera asimismo la causa determinante del éxito en el proyecto de agua rural del Banco Mundial en el Paraguay. Se ayudó a fortalecer una agencia gubernamental SENASA que tuvo la misión de promover en cada comunidad la creación de juntas y acordar con ellas contratos para la construcción y mantenimiento de los sistemas de agua. Se eligió esa alternativa que llevaría más tiempo en lugar de la de contratar una empresa externa que llevara adelante en corto plazo las construcciones. Los resultados convalidaron la elección: El proyecto excedió las expectativas. Las comunidades contribuyeron con el $21 \%$ de los costos totales de construcción (un $6 \%$ más que los estimados originales) y el sirve a 20.000 personas más que las originalmente estimadas. La operación y el mantenimiento son satisfactorios. Las juntas comunitarias están bien motivadas, manejan los sistemas satisfactoriamente, cumplen con los compromisos financieros, y tienen limitados problemas al recoger las contribuciones.

Dharam Gai (1989) llega a similares conclusiones a las de la investigación mencionada examinando nueve experiencias de participación popular en el tra. bajo con comunidades rurales pobres. Algunas se refieren a crédito para los pobres como la difundida del Grameen Bank, otras a organización de pequeños productores, grupos de autoayuda y ayuda mutua. Indica el investigador que en todos ellos, la efectividad es muy alta, y además es muy significativa la contribución al mejoramiento de la equidad. Resalta:

\footnotetext{
"Estas experiencias demuestran que una modalidad de desarrollo arraigada en organizaciones populares de participación, al mismo tiempo que permite la plena iniciativa individual y de grupos, promueve una distribución relativamente iqualitaria de los ingresos y el acceso a los servicios y medios comunes"
}

En América Latina numerosas experiencias en marcha indican que la participación comunitaria puede arrojar resultados fuera del alcance de otros tipos de abordajes en los campos más disímiles. Revisaremos brevemente tres de ellas, que son actualmente referencia internacional, llevadas a cabo en áreas muy diferentes: Villa EI Salvador en el Perú, las ferias de consumo familiar en Venezuela y el presupuesto municipal participativo en Porto Alegre, Brasil.

50.000 familias pobres se instalaron a comienzos de los 70 en un vasto arenal ubicado en las afueras de Lima, desprovisto de recursos de ninguna índole, y a distancia de vías de acceso. A ellos se fueron sumando otras familias marginales llegando a una población de 250.000 habitantes. Adoptaron un diseño urbanístico muy peculiar altamente descentralizado. El municipio está organiza- 
do por manzanas y cada grupo de manzanas tiene su propio parque central y espacios para la deliberación comunitaria, la recreación, y la cultura. Se organizaron en base a modelos altamente participativos, con directivos por grupo de manzanas, y más de 1000 espacios organizativos donde se desarrollan las actividades básicas de la Villa. En dos décadas, en condiciones socioeconómicas muy difíciles, levantaron con su esfuerzo comunitario gran parte de la infraestructura física. Construyeron 38.000 viviendas, 60 locales comunitarios, 64 centros educativos, 22 bibliotecas populares, 41 núcleos de servicios integrados de salud, educación, y recuperación nutricional, cuatro centros de salud, farmacias.

Su esfuerzo comunitario permitió lograr metas sociales de envergadura. El analfabetismo es $3,5 \%$ muy inferior a la media nacional. Asisten a primaria la casi totalidad de los niños y a secundaria un porcentaje mucho mayor a la media del país. La mortalidad infantil es marcadamente menor a la nacional, y lo mismo sucede con la mortalidad bruta. Hubo concreciones muy significativas en agricultura, y se estableció un parque industrial de microempresas. Se desenvolvió una densa vida productiva, social y cultural en medio de condiciones de adversidad económica muy intensas, con resultados muy diferentes a los de otras poblaciones marginales. La experiencia ha recibido continuos laudos mundiales. La UNESCO la premió como una de las más desafiantes experiencias de educación popular. Las Naciones Unidas la reconoció como promotora ejemplar de formas de vida comunitaria. España le concedió el premio Príncipe de Asturias como experiencia modelo de desarrollo social. En el Perú se le otorgó el Premio Nacional de Arquitectura por su diseño urbano. Recientemente, con apoyo de organizaciones de la sociedad civil, se ha transformado en uno de los primeros municipios de la región que ha incorporado la informática al servicio de la democracia. Se han instalado terminales públicos de computación y una red de televisión en circuito cerrado. Los habitantes reciben información sobre los temas a tratar por el Consejo Municipal por estas vías, y continuamente le hacen llegar por las mismas sus puntos de vista ${ }^{2}$.

La experiencia en su conjunto es desde ya de alta complejidad y ha tenido, como es inevitable, avances y retrocesos, pero sus logros son notables. La práctica permanente de la participación comunitaria ha sido una de sus bases principales. Uno de los resultados ha sido la elevación de la autoestima individual y colectiva, fuerza de enormes potenciali-

2 Puede verse al respecto "Villa El Salvador: Municipio Cibernético", Diario el Comercio, Lima, 23/junio/97. Respecto a Villa El Salvador en general, puede ampliarse información en: Carlos Franco. La experiencia de Villa El Salvador: del arenal a logros fundamentales a través de un modelo social de avanzada. Inciuido en Bernardo Kilksberg (comp.) "Pobreza. Un tema impostergable. Nuevas respuestas a nivel mundial" (Cuarta edic., Fondo de Cultura Económica, (1997). 
dades. Como describe un agudo analista de la experiencia Carlos Franco (1992):

\begin{abstract}
"Cuando se asiste con alguna frecuencia a reuniones de pobladores y se conversa con los "fundadores" de la comunidad o sus dirigentes, no resulta difícil advertir expresiones recurrentes de autoconfianza colectiva, certidumbres sobre la disposición de un poder organizado, una cierta creencia en las capacidades de la comunidad para proponerse objetivos y unirse para su logro".
\end{abstract}

Las Ferias de consumo familiar de Barquisimeto implican la incursión de la participación comunitaria en un terreno económico difícil, como es la comercialización de productos de consumo masivo. Son una red de 50 organizaciones donde participan productores agrícolas, cooperativas, asociaciones civiles, parroquias, que logran hacer llegar a la cuarta ciudad de Venezuela productos verdes y otros, a precios un $40 \%$ más barato que los del mercado. Las ferias compran a los productores sus cosechas a precios justos, $y$ las venden en los fines de semana a la población en ferias abiertas. Iniciadas con un capital de 4.000 dólares venden actualmente 25 millones de dólares anuales, y la repercusión de sus logros ha motivado el intento de replicarlas en otros estados. Cumplen múltiples funciones al mismo tiempo. Son: Organización de los productores agrícolas, apoyo a la producción popular, educación nutricional, formación en el trabajo, y una contribución clave al abaratamiento del costo de vida de los sectores populares. Han debido competir en el mercado con cadenas comerciales altamente especializadas $y$ con fuertes recursos financieros, y sin embargo, han logrado un posicionamiento creciente que las ha convertido en uno de los principales comercializadores de alimentos del país. En la base de sus logros se halla un sistema organizacional de participación activa. Identifican como bases de su organización las siguientes: reunión semanal de planificación y evaluación por grupos, rotación de responsabilidades, toma de decisiones por consenso, información para todos, disciplina y vigilancia colectiva, descentralización e integración, espacios de encuentro fuera de la tarea. Consideran que sus logros han tenido como pilares: una historia de formación de un capital humano y social, potenciar el capital social sobre el capital financiero, y unas formas de gestión novedosas. Se definen como un proyecto de vida, y no sólo productivo, orientado por valores como la solidaridad, la responsabilidad personal y de grupo, el amor al trabajo como medio para lograr el desarrollo personal y comunitario, la iniciativa personal, el respeto ${ }^{3}$.

En otro campo muy distinto a los anteriores se ha desenvuelto en América

3 Puede ampliarse al respecto en: "Las ferias de consumo familiar de Barquisimeto" (mineo), y Luis Gómez Calcaño "Las Ferias de Consumo Familiar del Estado Lara: una experiencia de organización participativa", Seminario: "Programa Sociales, Pobreza y Participación Ciudadana", BID, 1998. 
Latina en años recientes una experiencia que hoy es referencia internacional obligada, el presupuesto municipal participativo de Porto Alegre ${ }^{4}$. Con una población de 1.300 .000 habitantes y serios problemas sociales, el nuevo Alcalde electo de Porto Alegre, capital del estado de Río Grande del Brasil, abrió en 1989 un proceso por el cual las inversiones del presupuesto municipal serían decididas por toda la ciudadanía participativamente. Fuentes críticas vaticinaron una incierta suerte al propósito de hacer ingresar la participación a un área tan técnica. El Municipio estableció un sistema organizacional descentralizado que posibilitó el acceso masivo de sus habitantes a la toma de decisiones presupuestarias a través de su integración a grupos vecinales y niveles posteriores de representación de los mismos. Los habitantes recibieron por esta via información continua sobre el presupuesto y su ejecución, e identificaron sus propias prioridades. EI proceso les permitió según describe el BID (1997):

- expresar su comprensión de los problemas cruciales que enfrenta la ciudad,

- establecer prioridades de los problemas que merecen más inmediata atención,

- seleccionar las prioridades y generar soluciones prácticas,
- tener oportunidad de comparar con las soluciones creadas en otras regiones de la ciudad y en otros grupos de temas,

- decidir, con el apoyo de técnicos de la oficina del Alcalde, en invertir en los programas menos costosos y más factibles de atender,

- tomar la decisión definitiva sobre la aprobación, o no, del plan de inversiones, $y$

- revisar los éxitos y fracasos del programa de inversiones para mejorar sus criterios para el año siguiente.

La participación en el proceso ha ido creciendo y se estimaba que en 1995 más de 100.000 personas se vincularon a la creación del presupuesto de la ciudad. Los resultados refutaron los pronósticos escépticos, fueron más allá de lo esperado, y se han convertido en objeto de análisis internacional. La participación comunitaria masiva demostró ser un mecanismo más eficiente de asignación de recursos que los métodos tradicionales usuales. Los recursos fueron orientados hacia las prioridades reales de la población. Entre 1989 y 1995, entre otros aspectos, la cobertura de agua subió del $80 \%$ al $90 \%$ de la población, el sistema de alcantarillado se extendió del 46 al $74 \%$ de la población, las escuelas públicas matricularon el doble de niños, y se expandió el pavimento en los barrios pobres. Mejoró fuertemente el diseño y la gestión del presuel desarrollo redistributivo: el caso del presupuesto participativo en Porto Alegre, Brasil (19891998)" Seminario "Programas Sociales, Pobreza y Participación Ciudadana", BID; 1998. 
puesto, al recibirse los puntos de vista de la población, y hacerse totalmente transparente. Mas allá todavía la participación produjo un efecto "empoderamiento" de amplias proporciones. Como señala el BID: "El proceso participativo también ha tenido un enorme impacto en la habilidad de los ciudadanos para responder a los retos organizadamente, como comunidad, y en capacidad de trabajar en forma conjunta para mejorar la calidad de la administración pública y en consecuencia la calidad de la vida".

Los resultados de investigación expuestos, y los casos reseñados sumariamente a los que se pueden sumar muchos otros corroboran que la participación da resultados concretos y superiores a los esquemas tradicionales de "arriba hacia abajo". Las experiencias presentadas tienen, a pesar de su diversidad, dado que corresponden a realidades muy diferentes y han operado en campos muy variados, ciertas características comunes. En primer lugar, en todos los casos puede encontrarse que se intentó poner en marcha formas de participación "real", no "simulaciones de participación". La apelación no fue como se ha dado con tanta frecuencia a consultas erráticas o coyunturales, $o$ a recibir opiniones no tenidas en cuenta después, sino efectivamente se diseñaron modalidades organizacionales que facilitaron y estimularon la participación activa y continua. En segundo término, en todos los casos ha habido un respeto por aspectos como la historia, cultura e idiosincrasia de la población. No se "impusieron" formas de participación de laboratorio, sino que se intentó construir modalidades que fueran coherentes con esos aspectos. En tercer término, todas estas experiencias, que son de largo aliento, tuvieron como un marco subyacente un proyecto en términos de valores, de perfil de sociedades a lograr, de formas de convivencia diaria por las que se estaba optando.

¿Por qué la participación da resultados superiores? Ese es el objetivo de análisis de la siguiente tesis del trabajo.

\section{Segunda tesis. La participación tiene ventajas comparativas}

Los mejores resultados de los modelos participativos en el campo de los programas sociales, no son mágicos. Derivan de bases muy concretas. En general, los programas en esta materia, independientemente de sus metas especificas como bajar la deserción en primaria, mejorar las inmunizaciones, suministrar agua, prestar crédito a familias pobres, etc., tienen lo que se podría denominar "suprametas" que les son comunes y que enmarcan a las metas especificas. Se desea que los programas sean eficientes, es decir, hagan un uso optimizante de recursos usualmente escasos, que contribuyan a mejorar la equidad, punto crucial en América Latina, como se ha destacado actualmente la región más desigual del mundo, y que generen sostenibilidad, en tanto favorezcan la conformación de capacidades que fortalezcan la posibilidad de que la comunidad pueda seguir adelante con ellos en el tiempo.

Lograr este tipo de metas requiere un abordaje organizacional acorde con su particular estructura. Por otra parte, la 
tarea no estará cumplida maximizando una sola de las suprametas. Se debe tratar de lograr el mayor efecto de conjunto posible en los tres campos. Así, como ha sucedido en diversos casos si se hace un uso eficiente de recursos, y se alcanzan los objetivos, pero al mismo tiempo la metodología empleada es de carácter netamente vertical, los efectos pueden ser regresivos en términos de desarrollo de las capacidades de la comunidad, y las metas alcanzadas tendrán una vida limitada. Son usuales los proyectos de desarrollo social en donde se obtiene un nivel significativo de metas durante la duración del período del préstamo o la cooperación externa, pero al finalizar la misma, los logros retroceden rápidamente. Señala al respecto un análisis de la acción del Banco Mundial (Blustein, Washington Post, 1996): "evaluaciones internas indican que más de la mitad de sus proyectos, es incierto o improbable, sean "sostenibles". Ello significa que después que se han completado -un proceso que toma usualmente cinco o seis años- es posible que no sigan dando beneficios significativos a los paises receptores".

Tampoco la meta de equidad es de obtención lineal. No basta tener la intención de asignar recursos a través de los proyectos a grupos desfavorecidos. Si los modelos organizacionales empleados tienen características que sólo permiten el acceso real a dichos recursos a sectores de determinados niveles de calificación y capacitación previa, los programas pueden ser cooptados por tales sectores. Es frecuente el caso de programas para pobres, cuyas complejidades administrativas de acceso, llevan a que grupos de clase media se conviertan en sus principales beneficiarios.

Las dificultades reseñadas y otras identificables indican que debe haber una estrecha coherencia entre las metas de eficiencia, equidad y sostenibilidad, y el "estilo organizacional" empleado. Es esa la base práctica de la que surgen las ventajas comparativas de los modelos participativos genuinos. Sus rasgos estructurales son los más acordes con el logro combinado de las "suprametas".

En cada una de las etapas usuales de los programas: diseño, gestión, monitoreo, control, evaluación, la participación comunitaria añade "plus" prácticos, y limita los riesgos usuales.

En la elaboración del programa social, la comunidad puede ser la fuente mas precisa de detección de necesidades relevantes y de priorización de las mismas. Es quien más conocimiento cierto tiene sobre sus déficit y la urgencia relativa de los mismos. Asimismo puede hacer aportes decisivos sobre múltiples aspectos requeridos para un diseño exitoso, como las dificultades que pueden encontrarse en el plano cultural, y a su vez las "oportunidades" que pueden derivar de la cultura local.

Su integración a la gestión del programa logrará diversos efectos en términos de efectividad organizacional. Puede poner en movimiento la generación de ideas innovativas. Permitirá rescatar a favor del proyecto elementos de las tradiciones y la sabiduría acumulada por la comunidad que pueden ser aportes valiosos. Asegurará bases para "gerencia adaptativa". La experiencia de los programas sociales demuestra que ese es el tipo de gerencia más acorde a los mis- 
mos. Continuamente se presentan situaciones nuevas, en muchos casos inesperadas, y se necesitan respuestas gerenciales sobre la marcha. En gerencia adaptativa el momento del diseño y el de la acción deben acercarse al máximo. Para lograr resultados efectivos de la acción, el diseño debe reajustarse continuamente en base a los eventos emergentes. La comunidad puede posibilitar la gestión adaptativa suministrando en tiempo real continuos "feed backs" sobre qué está sucediendo en la realidad, e incluso agregando constantemente información que puede ayudar a evitar situaciones difíciles de manejar posteriormente.

En materia de control del buen funcionamiento del programa, y de prevención de la corrupción, el aporte de la participación comunitaria organizada puede ser insustituible. El control social obligará a la transparencia permanente, significará un seguro contra desvíos, permitirá tener idea a tiempo de desarrollos indeseables, a efectos de actuar sobre los mismos.

Finalmente, los jueces más indicados para evaluar los efectos reales de los programas sociales son sus destinatarios. Las metodologías modernas de evaluación participativa, y de investigación acción permiten que la comunidad de modo orgánico indique resultados efectivamente obtenidos, deficiencias, efectos inesperados favorables y desfavorables, y elementos claves para diseños futuros.

No utilizar los modelos participativos significará "costos de oportunidad" en todos los aspectos organizativos planteados. Pero además, favorecerá la generación de "costos directos" que atentarán contra el cumplimiento de las metas, como los siguientes identificados por el Grupo de Desarrollo Participativo del Banco Mundial (1994):

- una falta de apoyo y de sentido de propiedad que impide el aprovechamiento de los servicios, reduce la continuidad del beneficio y limita la recuperación de los costos del proyecto.

- un sentido de indiferencia y dependencia del Estado, donde los ciudadanos ven que tienen poca o ninguna voz en su propio desarrollo.

- malestar y resentimiento cuando los proyectos o políticas son impuestos; y limitación del aprendizaje y la creación de nuevas alternativas por parte de los actores claves.

- costos financieros, de tiempo y oportunidad adecuada si el Banco y los actores claves no cambian, se identifican mutuamente y se comprometan unos con otros.

- dificultad para asegurar que los actores claves y sus prioridades reales están expresados apropiadamente por las personas que los representan.

- el riesgo de ahondar diferencias y conflictos preexistentes entre subgrupos de interesados con diferentes prioridades e intereses.

- generar expectativas imposibles de cumplir, $y$

- las élites poderosas y más organizadas pueden tomar el poder y excluir a la gente de escasos recursos y a los grupos marginados.

Todos los "plus" de la participación comunitaria señalados y otros añadibles aportan fuertemente a la eficiencia organizacional. Pero su efecto combinado va mucho más allá de ello. Tienen impactos 
extensos y profundos en materia de sostenibilidad y equidad.

En cuanto a la sostenibilidad, al crearse condiciones favorables para ello a través de la participación, la comunidad puede desarrollar el sentimiento de "ownership", de propiedad del proyecto, hacerlo realmente suyo. Ello movilizará sus energías y esfuerzos para que el mismo avance, y creará una conciencia de protección de sus concreciones. La participación asimismo posibilitará condiciones para que la comunidad aprenda, se ejercite en el planeamiento y la gestión, y vea crecer sus capacidades. Se fortalecerấ entonces su posibilidad de sostener el proyecto.

Todos los elementos mencionados potenciarán la autoestima individual y colectiva. Ello puede desencadenar energías y capacidades latentes en gran escala.

La experiencia permite constatar el valor para la sostenibilidad del abordaje participatorio. A partir de ella resalta la Organización para la Cooperación y el Desarrollo Económico (OECD, 1993); "Para que el desarrollo sea sostenible, las personas de los países interesados deben ser los "dueños" de sus políticas y programas de desarrollo".

Los riesgos en materia de que los programas no mejoren la equidad pueden ser considerables. En la visión de la Agencia Canadiense para el Desarrollo Internacional (CIDA, 1994): "El beneficio de los proyectos de desarrollo llegaba generalmente mas a los que estaban en mejores condiciones, a los ubicados en áreas accesibles y a los que tenian mejor acceso a la información".
La participación comunitaria en todas las etapas de los proyectos, ese pensar la lógica del proyecto desde las percepciones y la cultura de los pobres, los acercará mucho más a sus realidades y reducirá riesgos como los señalados.

Al mismo tiempo, la participación en sí como proceso social cambia a sus mismos actores. Potencia a los grupos desfavorecidos, hace crecer sus propias capacidades, y contribuye a su articulación. Todos esos elementos los colocan en mejor situación para luchar por sus derechos e influir de modo efectivo.

Este conjunto de ventajas comparativas son las que operan detrás de la superioridad observable en las experiencias con participación respecto a los modelos organizacionales de corte tradicional de tipo jerárquico o paternalista.

Las ventajas son reconocidas como tales actualmente por un consenso muy amplio en otros campos organizacionales, como se podrá observar en la tesis siguiente.

\section{Tercera tesis. \\ La participación es un núcleo central de la gerencia del año 2000}

La revalorización de la participación en el campo social se inscribe en un proceso mas generalizado donde están cambiando fuertemente las percepciones respecto a los aportes de la participación a la gerencia.

Está en plena marcha a fines del siglo XX un cambio de paradigma de extensas implicancias en cómo obtener eficiencia en las organizaciones. Las ideas que dominaron la gerencia durante casi 
todo este siglo, y siguen ejercitando una influencia determinante en América Latina, asociaban gerencia de calidad, con aspectos como organigramas precisos, división de funciones, manuales de cargos, descripción detallada, procedimientos, formularios. La visión era que "ordenando" formalmente la organización, poniendo bajo control de las normas y procesos la mayor parte de su funcionamiento, se obtendrian resultados exitosos.

$\mathrm{El}$ análisis cientifico de algunas de las organizaciones con mejores resultados actuales indica que los estilos gerenciales que han adoptado se hallan totalmente distantes del paradigma tradicional. Estudios pioneros como los de Kotter en Harvard (1989) y Mintzberg (1996) en la Universidad McGill en Canadá, coinciden en identificar que el éxito se asocia en factores como capacidades para el análisis sistemático del contexto y sus tendencias, detección de los problemas estratégicos, comunicaciones activas, horizontalidad, participación, potenciación de las capacidades de la organización, construcción de redes de contactos, y otros semejantes. Se ha descripto la transición paradigmática en desarrollo como el "paso de la administración a la gerencia"s.

Como ha sucedido normalmente en la historia, los cambios de paradigma no se dan a instancias exclusivamente de personas. Tienen que ver con modificaciones profundas en la realidad, que plantean nuevas demandas. Efectivamente, ha habido en las décadas recientes transformaciones estructurales en el contexto histórico que plantearon exigencias cualitativamente diferentes a la gerencia de organizaciones. Los cambios simultáneos en dimensiones fundamentales de la realidad, como las tecnologías, la geopolítica, la geoeconomía, y otros producidos en períodos cortos y en medio de un sistema mundial cada vez más interconectado, generaron un contexto de umbrales de complejidad inéditos. Uno de sus rasgos centrales es grados de incertidumbre pronunciados. Los impactos sobre la gerencia son múltiples. Entre ellos, gran parte de las variables del contexto pueden afectar en cualquier momento a la mayor parte de las organizaciones. Sus contextos son ahora, como se dice en gestión estratégica, "un mundo de entrometidos" donde variables intrusas de todo orden aparecen sorpresivamente e influyen. Asimismo, el tiempo ha mutado sus características. En gerencia tradicional se entrenaba a proyectar las realidades pasadas, y a tomar decisiones en base a esas proyecciones. Se extrapolaban cifras presupuestarias, participaciones en el mercado, etc. Actualmente, en una época donde las tasas de cambio de la realidad son ultraaceleradas, el pasado puede ser una guía engañosa. El presente difiere radicalmente del pasado. A su vez, el futuro no se halla a gran distancia, como sucedia antes. El presente se transforma muy velozmente, convirtiéndose rápidamente en futuro. Las fronte-

5 El autor analiza detalladamente el tema en su obra "El pensamiento organizativo: de los dogmas a un nuevo paradigma gerencial" (13 edic., Editorial Norma, 1994). 
ras entre ambos son cada vez más cercanas. La gerencia no puede apoyarse en la proyección del pasado, ni en cuidadosas planificaciones de mediano y largo plazo. Tiene que ser fuertemente adaptativa, y tener gran capacidad de innovar.

El medio sumariamente descripto exige otro tipo de diseños organizacionales, de estilos gerenciales, y de habilidades en los miembros de la organización. Las organizaciones que han logrado desenvolverlos están a la vanguardia en logros en diversos campos. La imagen ideal de la organización mutó. No es la de una organización rigurosamente ordena-

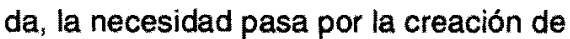
"organizaciones inteligentes", con capacidad de tener una relación estrecha con el contexto, entender las "señales de la realidad" y actuar en consecuencia. Para ello deben ser necesariamente "organizaciones que aprenden". Entre sus capacidades esenciales estará la de saber "gerenciar conocimientos". Este tipo de organizaciones no son viables sin un personal comprometido. La inteligencia, el aprendizaje, la administración del conocimiento, la innovación, no se hallan al alcance de una persona por mayores que sean sus cualidades. Sólo pueden ser generadas desde el conjunto del personal, operando a través de equipos de trabajo. Peter Ducker (1993) plantea agudamente: "El líder del pasado era una persona que sabía cómo ordenar. El del futuro tiene que saber cómo preguntar". Necesita imprescindiblemente de los otros. Como resalta Goldsmith (1996), entre las habilidades de los ejecutivos exitosos se hallan ahora las de escuchar, hacer "feed back" continuo, no caer en el usual sesgo de las estructuras jerárquicas tradicionales de "matar" al que dice la verdad, sino por el contrario estimular, reflexionar.

El modelo deseado para el 2000 es el de: organizaciones inteligentes, que aprenden, adaptativas, innovadoras. Buscando caminos para construirlas, gerentes, expertos e investigadores Ilegaron permanentemente en los últimos años a la participación. Estudios pioneros como los de Tannenbaun (1974) ya arrojaban evidencias al respecto. Analizando empresas jerárquicas y participativas en diversos países se observaron significativas correlaciones entre altos grados de participación y mayores niveles de satisfacción, mayor motivación laboral, e incluso menor frecuencia de síntomas de úlcera. Walton (1995) indica que desde los 70 diversas empresas emprendieron lo que llama "la estrategia del compromiso" tratando de lograr el involucramiento activo de su personal. Menciona entre ellas plantas de la General Foods, General Motors, Procter and Gamble, Cummings Engine. Los beneficios para la productividad eran muy claros. En Japón surgieron los circulos de calidad, basados en la idea de capitalizar los aportes que en cada sector de la empresa podian hacer los operarios al mejoramiento de las tareas. Se realizaban en horas de trabajo, eran alentados fuertemente, tenian incentivos. Se estimó que aportaron cerca del $60 \%$ de las mejoras de productividad de la empresa japonesa durante un extenso periodo de tiempo. Constituian una forma básica de participación. A fines de siglo la participación es convocada gerencialmente desde llamados de orden sofisticado. Así se plantea que un motor de la organización es la "visión compartida". Peter Senge (1992) la considera un 
instrumento eje para la productividad. Crea una sensación de vínculo común, da coherencia a las actividades, inspira. Estudiando equipos con alto desempeño, Maslow (1965) ya habia anticipado que uno de sus rasgos esenciales era la visión compartida.

En esos equipos de excepción anotó: "La tarea ya no estaba separada del yo... sino que él se identificaba tanto con la tarea, que ya no se podía definir el verdadero yo sin incluir esa tarea".

Se requiere, asimismo, la participación para crear un ambiente altamente deseado hoy en gerencia avanzada: un clima de confianza. Las mediciones indican fuertes correlaciones entre clima de confianza y rendimiento $y$, al revés, entre percepción del personal de que se desconfía de él, y reducción del rendimiento. El esquema básico de la administración tradicional de corte vertical está fundado en la presunción de que se debe desconfiar del personal, y ello es captado por el mismo. A su vez la confianza tiene doble vía. El personal debe sentir que puede confiar en la organización. Que aspectos como, por ejemplo, los ascensos, y el acceso a oportunidades, estarán regulados por criterios objetivos.

La creación de "confianza" necesita participación. Ese es su hábitat natural.

Por otra parte, se aspira hoy a una alta tasa de innovación. Sin ella en los mercados actuales, competitividad. Las investigaciones demuestran que la tasa de innovación es mayor en los trabajos en equipos interdepartamentales, lo que significa estructuras horizontalizadas. También indican en forma consistente que algunas de las innovaciones más importantes en el mundo organizacional en los últi- mos años se han dado en el marco de lo que llaman "grupos calientes". Se trata de grupos reducidos, autogestionados, con un desafío importante, cabalmente participativos (Leavitt, Lipman-Blumen, 1995).

Diversos analistas describen el panorama de la organización del futuro con visiones que prevén altos contenidos participativos. Para Hackman (1986) "Las organizaciones en el futuro se basarán fuertemente en la autogestión de sus miembros". Peters (1988) dice que: "las organizaciones utilizan equipos multifuncionales y organizan cada función con 10 a 30 personas en grupos autogestionados". Para Wilpert (1984) "La participación en el trabajo organizacional será un tema central... en todos los países industrializados o en industrialización".

La búsqueda de eficiencia apelando a la participación forma parte básica también de experiencias de vanguardia en la gestión pública. Kernaghan (1992) reseña la amplia experiencia de los Gobiernos Canadienses. La idea clave de diversas experiencias exitosas que se están realizando con participación de los funcionarios en los servicios públicos canadienses "es liberar el talento de los empleados cambiando la cultura de la organización por una que involucre y faculte mas y cambiando la estructura de la organización por medio del uso de grupos de trabajo facultados". La participación que se busca no es sólo la de los empleados individualmente, sino en equipo reestructurándose así toda la conformación de la organización tradicional. En base a 68 casos de experiencias participativas en el sector público canadiense en los últimos años el autor elabora una vivida reconstrucción de cómo evolucionaron los pro- 
cesos participatorios que por su agudeza transcribimos integralmente a continuación:

"Al comienzo del proceso la organizacion tiene las siguientes caracteristicas: la mayoria de los gerentes operan siguiendo el estilo de mando y cumplimiento pero por lo menos algunos apoyan la participación de los empleados y el trabajo en grupo, un pequeño porcentaje de empleados participa en actividades de grupo, sólo existen planes generales no específicos para incrementar la participación de los empleados, la forma y el número de sugerencias de los empleados han sido relativamente estables durante los últimos años; y las mejoras al ambiente de la organización y a las prácticas de manejo de recursos humanos resultan de la sugerencias y quejas de los empleados. Las etapas siguientes del proceso muestran un paso gradual hacia una organización facultada. Hacia el final de este proceso, se ha logrado una transformación notable". Según observa el investigador, al transformar la organización de un modelo jerárquico tradicional, a un modelo participativo, se da paso a una organización con las siguientes características:

- "La administración usa métodos inno. vativos $y$ efectivos para incrementar la participación de los empleados y el trabajo en equipo, existe un alto nivel de confianza y respeto entre los empleados, entre los gerentes y entre empleados y gerentes.

- Surge una cooperación entre los grupos que realizan diferentes funciones en toda la organización para satisfacer las necesidades de los clientes de una manera más efectiva.
- Las tendencias hacia la participación en equipo y otras formas de participación de los empleados permiten que los empleados hagan más sugerencias y aumente el número de sugerencias aceptadas.

- Los empleados se sienten fuertemente facultados, existe un sentimiento de propiedad grupal sobre los procesos de trabajo los empleados muestran un orgullo personal por la calidad del trabajo y el sindicato y la administración cooperan para mejorar la calidad

- El poder, las retribuciones, la información, y el conocimiento se llevan hasta los niveles más bajos factibles; el facultamiento de los empleados conduce a una nivelación sustancial de la organización.

- Las mejoras que resultan de la partjcipación de los empleados se hacen evidentes en los sistemas, procesos, productos y servicios.

- Un proceso de encuesta formal regular determina los niveles de satisfacción de los empleados, se emprenden acciones de seguímiento para mejorar las prácticas de manejo de recursos humanos y los planes futuros determinan cómo sostener el momento y el entusiasmo".

Las experiencias participativas canadienses arrojaron múltiples beneficios. Entre ellos: mejor productividad, moral más alta, reducción de costos, mejor servicio a los clientes, más innovación y creatividad, reducción en el ausentismoy la rotación de personal. Una ventaja adicional de las organizaciones abiertas a la participación, es que demuestra tener 
una mayor capacidad de atracción de personal calificado y capaz. El proyecto laboral global que brindan, les da superioridad competitiva en el reclutamiento respecto a organizaciones de corte tradicional.

Schelp (1988) refiere un interesante caso en el servicio público en Suecia. El enfoque participatorio fue aplicado en profundidad a la comunidad en la prevención de accidentes en municipios rurales. Se hizo tomar conciencia a la comunidad de los resultados de salud en esta área no dependían de los servicios de salud sino, sobre todo, de la acción preventiva conjunta de la misma comunidad realizada incluso en los hogares. Las principales causas de este tipo de accidentes no eran prevenibles desde afuera de la comunidad sino sólo desde su interior. Se crearon grupos de trabajo comunitarios que asumieron responsabilidades crecientes en la labor preventiva a los que se dio pleno apoyo, y se realizó desde ellos una tarea de difusión amplia sobre los patrones de accidentes mas frecuentes, y las politicas necesarias para prevenirlos. Al cumplirse tres años de la experiencia la tasa de accidentes había decrecido en un $30 \%$. Por otra parte, el número de miembros de la comunidad interesados en participar ascendió considerablemente. En la estrategia empleada, el sector público transfirí a la comunidad conocimientos y experiencia. La misma a través de sus organizaciones básicas: ONGs, empresas sindicatos, individuos, asumió el peso de la acción.

Extrayendo conclusiones en este campo Mintzberg (1996) llama la atención sobre que en definitiva los servicios en salud y educación "nunca pueden ser mejores que las personas que los sumi- nistran". Se hace necesario "liberar" el potencial de esas personas. La participación claramente aporta a ello.

Como se observa, tanto en el campo gerencia empresarial como en el público, las indicaciones hacia la participación tienen fuerza creciente. Participación es hoy una estrategia maestra de la gerencia de excelencia.

Frente a los resultados que da la participación comunitaria, sus ventajas comparativas, y su legitimidad gerencia, ¿cómo se explica su limitado avance en la región?

A dicho problema está dedicada la siguiente tesis.

\section{Cuarta tesis. La participación enfrenta fuertes resistencias e intereses}

En el "discurso" la participación ha triunfado en América Latina. Se escuchan permanentemente desde los más altos niveles gubernamentales, y de grupos de gran peso en la sociedad, referencias a la necesidad de incrementar la participación, a su deseabilidad para una sociedad democrática, a su tradición histórica en cada sociedad. A diferencia de décadas cercanas, casi no se escuchan voces que explícitamente se oponga a la participación. Sin embargo, la realidad no pasa solamente por el discurso. En los hechos, los avances en participación comunitaria muestran una gran brecha con el declaracionismo al respecto. Las investigaciones que se han internado en la práctica de la participación han encontrado con frecuencia, llamados a participar que no se plasman en apertura efectiva de puertas, experiencias iniciadas con 
amplias promesas pero que se quedan en el "título" inicial, frustraciones pronunciadas de numerosas comunidades.

La brecha tiene explicaciones. La participación comunitaria es en definitiva un proceso que implica profundos cambios sociales. Como tal es esperable que genere resistencias, y que al vulnerar intereses instalados los mismos desarroIlen estrategias de obstaculización.

Es fundamental poco a poco descubrir de dónde provienen las principales trabas a su avance, para poder diseñar políticas adecuadas de superación de las mismas.

Entre ellas, en nómina no taxativa, se hallan las que sumariamente se presentan a continuación.

\section{A. El eficientismo cortoplazista}

Una resistencia primaria a la participación es la de cuestionarla en términos de costos, y tiempo. El razonamiento explicito plantea que montar un proyecto con componentes participativos implica toda una serie de operaciones adicionales a su mera ejecución directa, que significan costos económicos. Al mismo tiempo se resalta que los tiempos de implementación se extenderán inevitablemente por la intervención de los actores comunitarios. Generará costos y alargará los plazos.

E| razonamiento demuestra pronunciadas debilidades cuando se sugiere un análisis que exceda el cortoplacismo. En una primera impresión efectivamente en muchos proyectos habrá nuevos costos por la participación, y los plazos serán más extensos. ¿Pero cuál es el impacto de estas "cargas adicionales" en el mediano y largo plazo? La alternativa real no es entre efectividad a corto plazo, y efectividad con mayores costos a largo plazo.

La evidencia ha demostrado sistemáticamente que los logros cortoplacistas tienen desventajas pronunciadas. Por lo pronto, como se ha destacado, una de las metas centrales en proyecto sociales, la sostenibilidad del proyecto, resiente agudamente con esos planteos, las evaluaciones internas practicadas al respecto por organizaciones como el Banco Mundial son casi terminantes. Un porcentaje significativo de proyectos, evaluados con indicadores apropiados, no pasan al test de sostenibilidad. La actividad se desarrolló de tal modo que terminada la cooperación del organismo externo a la comunidad, no han quedado bases para que la comunidad se sienta estimulada o esté capacitada para seguir sosteniendo el proyecto. La efectividad de corto plazo se transforma alli en altos niveles de inefectividad a medio y largo plazo.

Por otra parte el razonamiento eficientista, implica cuantiosos "costos de oportunidad". Los extensos beneficios potenciales derivados de la participación comunitaria y reseñados en las secciones anteriores no se producirán. Véase por ejemplo entre muchos otros el caso del proyecto PRODEL en Nicaragua (Stein, 1998). Su objetivo es movilizar pequeños proyectos de infraestructura y equipamiento urbano. Se opto por realizarlo bajo un modelo de cogestión con la comunidad. Las evaluaciones realizadas indican que con ella los costos directos de construcción y mantenimiento preventivo de estas obras fueron hasta un $20 \%$ inferiores al costo de proyectos similares ejecutados por los gobiernos locales sin participación comunitaria. Entre otros aspec- 
tos la ciudadanía aportó al proyecto 132.000 dias de trabajo voluntario.

\section{B. El reduccionismo economicista}

Otra línea de razonamiento coherente con la anterior percibe todo el tema del diseño y ejecución de programas sociales desde categorías de análisis puramente económicas. Las relaciones que importan son de costo/beneficio medido en términos económicos. Los actores se hallarían motivados por cálculos microeconómico puros, y persiguen básicamente la maximización de su interés personal. Lograr que produzcan, sería un tema de meros "incentivos materiales". Las evaluaciones desde este enfoque sólo perciben los productos medibles con unidades económicas. Muchos de los aspectos de la participación comunitaria no ingresan por tanto en este marco de ubicación frente a la realidad. Ella genera productos como el ascenso de la autoestima, y la confianza en las fuerzas de la comunidad que escapan a este razonamiento. Las motivaciones a las que apela como responsabilidad colectiva, visión compartida, valores de solidaridad, no tienen que ver con los incentivos economicistas. Las evaluaciones no tienen en cuenta los avances en aspectos como cohesión social, clima de confianza, y grado de organización.

Al desconocer todos estos factores el economicismo priva a la participación de "legitimidad". Es una especie de ejercicio de personas poco prácticas, o soñadoras sin conexión con la realidad. Sin embargo, los hechos indican lo contrario. Los factores excluidos, forman parte central de la naturaleza misma del ser humano. Cuando se niegan hay sensación de opresión, y las personas se resisten a aportar utilizando múltiples estrategias. Cuando facilitan, en cambio, dichos factores, pueden ser un motor poderoso de productividad.

Amartya Sen (1987) realiza sugerentes anotaciones sobre los errores que implica el economicismo. Señala que "la exclusión de todas las motivaciones y valoraciones diferentes de las extremadamente estrechas del interés personal es difícil de justificar en términos de valor predictivo, y parece tener también un soporte empírico dudoso". Los seres humanos tienen otros tipos de comportamiento -indica-éticamente influidos, entre ellos: sienten simpatía por otros, se comprometen con ciertas reglas de conducta, tienen lealtades, tienen interdependencias. "Los fríos tipos racionales llenan nuestros libros de texto pero el mundo es más rico". Los seres humanos hacen errores, experimentan, están confusos, hay Hamiets, Mcbeths, Lears, Otellos.

Sen se pregunta: "Es extraordinario que la economía haya evolucionado por una vía que caracteriza la motivación humana de un modo tan estrecho; es extraordinario porque se supone que la economía está preocupada por la gente real. Es diffcil creer que esa gente esté completamente no afectada por el tipo de examinación que plantea la pregunta socrática "cómo debería uno vivir". ¿Puede la gente que la economía estudia realmente no estar alectada por esta resonante cuestión, y seguir exclusivamente el rudimentario razonamiento duro que les atribuye la economía moderna?".

\section{El predominio de la cultura organizacional formal}

Un paradigma antes reseñado ha dominado el pensamiento organizativo 
en la región, la visión formalista. Para ella el orden, la jerarquía, el mando, los procesos formalmente regulados, y una percepción verticalista y autoritaria de la organización, son las claves de la eficiencia. Como lo detectara Robert Merton, en este enfoque el orden, que es un medio, tiende a transformarse en un fin en sí mismo. En este tipo de organizaciones se produce una traslación de valor de los fines a las rutinas. El cumplimiento de la rutina está por encima de lo sustantivo.

Esa cultura lee como "heterodoxa" e intolerable la participación, que está basada en la cooperación, la horizontalidad, la flexibilidad, la gerencia adaptativa, la visión clara de cuáles son los fines y la subordinación a ellos de los procesos organizativos. El choque entre ambas culturas es inevitable. Cuando se encomienda a organizaciones de tradición burocrática y vertical poner en marcha proyectos participativos, las resistencias serán innúmeras, y se expresarán por múltiples vías. Pondrán obstáculos infinitos, asfixiarán a fuerza de rutinas los intentos, cerrarán las puertas a las iniciativas, desmotivarán continuamente a los actores comunitarios. Estarán en definitiva esperando inconscientemente el fracaso de la experiencia participatoria para convalidar desde él su propio modelo burocrático formal.

\section{La subestimación de los pobres}

En diversas oportunidades sectores directivos y profesionales de las organizaciones que deben llevar a cabo proyectos porvias participatorias, tienen una concepción desvalorizante de las capacidades de las comunidades pobres. Creen que serán incapaces de integrarse a los procesos de diseño, gestión, control, y evaluación. Que no pueden aportar mayormente por su debilidad educativa $y$ cultural. Que necesitarán periodos muy largos para salir de su pobreza. Que sus liderazgos son primitivos, que sus tradiciones son atrasadas, que su saber acumulado es una carga.

Cuando se parte de una concepción de este orden se desconfiará de las comunidades en todas las etapas del proceso, se le limitarán las opciones reales para participar, se tendrá un sesgo pronunciado a sustituir su participación por ordenes de "arriba hacia abajo" para hacer "funcionar" las cosas. Asimismo la subvaloración será captada rápidamente por la comunidad, y ello creará una distancia infranqueable entre elía y los encargados de promover su participación. Todas estas condiciones crearán una situación en donde la participación estará condenada a fracasar. Después con frecuencia aparece en las "elites ilustradas" que condujeron la experiencias la coartada racionalizadora. Argumentarán que las comunidades no tenian interés en participar, y por eso la experiencia no operó. En realidad ellos crearon fuertes incentivos para que perdieran el interés.

La idea de "capital social" de creciente difusión, rompe categóricamente con estos mitos sobre las comunidades pobres. Una comunidad puede carecer de recursos económicos, pero siempre tiene capital social. Las comunidades pobres tienen normalmente todos los elementos constituyentes del capital social: valores compartidos, cultura, tradiciones, sabiduría acumulada, redes de solidaridad, expectativas de comportamiento recíproco. Cuando logran movilizar ese ca- 
pital social, los resultados pueden ser tan importantes como los observados en este trabajo en Villa EI Salvador del Perú, o las ferias de consumo familiar de Venezuela. Por otra parte como anotara Albert Hirschman (1984) a diferencia de otras formas de capital, el capital social es el único que aumenta con su uso

\section{E. La tendencia a la manipulación de la comunidad}

Un poderoso obstáculo al avance de la participación se halla en los intentos reiterados en la realidad latinoamericana de "coparla" para fines de determinados grupos. El clientelismo es una de las formas favoritas que adopta la manipulación. Alli el discurso ofrece promesas muy amplias de participación para ganar apoyos temporarios. Luego las realidades son muy pobres en participación real. Incluso sistemáticamente en los intentos manipulatorios se trata de relegar a los lideres auténticos de la comunidad y de impedir que surjan líderes genuinos. Se procura asimismo crear "líderes a dedo" que puedan ser en definitiva un punto de apoyo para el proyecto manipulatorio. Cuando la comunidad percibe las intenciones reales, se produce un enorme efecto de frustración. Los efectos son graves. No sólo la comunidad resistiéndose dejará de participar, y la experiencia fracasará, sino que habrá quedado fuertemente predispuesta en contra de cualquier intento posterior aun cuando sea genuino.

\section{F. El problema del poder}

La investigación antes mencionada de Narayan sobre los proyectos rurales de dotación de agua, constata la presen- cia como obstáculos a la participación de muchos de los mencionados. Indica que entre los problemas identificados se hallaron: la resistencia a dar el control sobre los detalles de la implementación, la falta de incentivos para una orientación hacia la comunidad, la falta de interés en invertir en desarrollo de las capacidades de la comunidad.

A estos y otros obstáculos mencionables, corresponde sumarles un obstáculo formidable muchas veces subyacente detrás de los anteriores.

Mary Racelis (1994) indica que un eje central en participación es "el conferimiento de poder al pueblo en lugar de perpetuar las relaciones generadores de dependencia tan caracteristicas de los enfoques de la cima a la base", La idea es compartir realmente el poder. Esto es lo que sucedió en la exitosa experiencia del presupuesto municipal participativo de Porto Alegre. Según refiere Zander Navarro (1998) no sólo redistribuyó los fondos públicos de un modo más equitativo instalando un patrón más justo que priorizóa los pobres, sino estableció un nuevo marco de relaciones políticas. La Comunidad electivamente fue investida del poder de decidir, y pusieron a su disposición mecanismos concretos de deliberación para ejercerlo que ella misma fue enriqueciendo con su práctica. El investigador se pregunta si esa experiencia es trasladable a otros municipios. Su respuesta destaca que "el requisito más importante y decisivo a tenerse en cuenta es que las autoridades locales deben tener la firme voluntad política de compartir partes de su poder con sus constituyentes".

Un obstáculo fundamental en el camino a la movilización de la participación 
es si existe una voluntad en ese orden. Si hay disposición realmente a compartir el poder.

A veces ella no existe. El proyecto que se está llevando a cabo está ligado a ciertos fines de algunos sectores, y dar participación real podría obstaculizarlo. En otras ocasiones, el cálculo es el que disminuiría el poder que tendrían las autoridades.

Sin embargo, con participación los efectos podrían ser muy diferentes. En alta gerencia el llamado de investigadores como John Kotter de la Universidad de Harvard a organizaciones empresariales más abiertas a la influencia de sus integrantes despertó inicialmente muy fuertes resistencias en el liderazgo empresarial tradicional. Pero después de años de lanzado, el autor indica que la experiencia real fue en sentido opuesto. Quienes compartieron el poder organizacional, actuaron de ese modo en aspectos claves de su organización: incrementaron la innovatividad y la productividad, y aumentaron entonces el "poder total disponible" de la misma. Quienes se encerraron y no aceptaron compartir, fueron los dueños absolutos de organizaciones cada vez menos competitivas, por lo tanto de un "poder total" en reducción.

Experiencias como las de Porto Alegre y otras sugieren que procesos semejantes se dan en el campo de la participación comunitaria. Las autoridades municipales que desarrollaron en Porto Alegre un proyecto genuinamente participativo recibieron un apoyo creciente y cada vez más generalizado de toda la población de la ciudad, que percibió que toda la ciudad mejoraba. Sus bases reales de poder no disminuyeron compartiéndolo, sino aumentaron, y fueron reelectas en varias oportunidades.

¿Cómo enfrentar las importantes resistencias y obstáculos reseñados a la participación y otros agregables?

\section{Quinta tesis. Se requieren políticas y estrategias orgánicas y activas para hacer avanzar la participación}

Los avances en participación comunitaria sufren permanentemente el embate de obstáculos y resistencias como los señalados. Pero existen tambiến en los procesos históricos actuales de la región importantes fuerzas en pro de dichos avances. Los trascendentales progresos realizados por la región en el campo de la democratización crean un marco objetivo de condiciones proparticipación.

En la América Latina actual hay una vigorosa presión de la población porque la democracia conseguida a través de largas luchas adquiera características cada vez más activas. Se aspira a reemplazar la "democracia pasiva" por una "democracia inteligente" donde el ciudadano esté ampliamente informado, tenga múltiples canales para hacer llegar continuamente sus puntos de vista-no sólo la elección cada tantos años de las autoridades máximas- y ejerza una influencia real constante sobre la gestión de los as untos públicos. Se están desarrollando positivos y crecientes procesos de fortalecimiento de la sociedad civil. Aumenta a diario el nümero de organizaciones de base, mejora su capacidad de acción, se está enriqueciendo el tejido social.

Todo este medio ambiente en cambio crea actitudes y percepciones cultura- 
les que ven a la participación de la comunidad como una de las vias principales para activar la democracia en los hechos concretos.

Junto a ello, las urgencias sociales latinoamericanas son extensas, y profundas. La región esta llegando al año 2000 con amplios sectores de la población sin agua potable, $y$ sin instalaciones sanitarias mínimas. Con una amplia población desnutrida, lo que va a significar severas consecuencias. Se ha estimado así que una tercera parte de los niños de Centroamérica menores de 5 años de edad presentan una talla menor a la que debieran. El $50 \%$ de los niños de la región no completan la escuela primaria, desertan antes de finalizar seis grados. La repetición es del $50 \%$ en el primer grado, y de $30 \%$ en cada uno de los grados posteriores. Ello produce, según indica Puryear (1998), que un niño de la región promedio permanece siete años en la escuela primaria y completa en ellos sólo cuatro grados. Las tasas de desocupación abierta son muy elevadas y las de desocupación juvenil aun mayores. Ha crecido aceleradamente la violencia urbana. La unidad familiar esta agobiada por el peso de la pobreza, y se destruyen numerosas familias.

Encarar los dificiles problemas señalados requerirá políticas públicas renovadas, donde asoma la necesidad de concebir diseños de políticas que articulen estrechamente lo económico y lo social, y dar alta prioridad a agresivas políticas sociales. La instrumentación de nuevas políticas y programas requiere imaginación gerencia. Se necesitan modelos no tradicionales de mayor efectividad. Allí la participación comunitaria, como se ilustró en las secciones previas del trabajo, da resultados, y tiene ventajas comparativas.

Estas y otras demandas y fuerzas proparticipación deben ser movilizadas para afrontar las resistencias y obstáculos. Se requiere a tal fin diseñar y poner en práctica políticas y estrategias apropiadas para dar la "pelea por participación". Entre ellas:

a) Hay una vasta tarea de investigación a realizar en la materia. Es necesario apuntalar la acción con estudios sistemáticos sobre los factores a tener en cuenta para aprovechar el potencial de la capacitación, y poder solucionar los problemas inevitables que aparecerán en sus procesos de ejecución. Así en la investigación realizada por el Banco Mundial sobre proyectos rurales de dotación de agua (Narayan 1994) se concluye del análisis de los 121 proyectos examinados que entre los factores favorables al éxito de la participación se hallan los siguientes:

i. en cuanto a los beneficiarios de los proyectos:

- obtener el compromiso de los beneficiarios previamente a la implementación del proyecto;

- incidir en el grado de organización de los beneficiarios.

ii. en cuanto las agencias ejecutoras de los proyectos:

- hacer del avance de la participación una meta central de sus proyectos;

- consiguientemente debe haber un monitoreo sistemático de cómo están adelantado las "metas de participación comunitaria", 
- crear incentivos y reconocimientos por iniciativas de miembros de la organización que aporten al avance de la participación;

- la agencia debe tener fuerte orientación a aprovechar el conocimiento de la comunidad;

- debe asimismo orientarse consistentemente a invertir en la capacitación de la comunidad.

Estudios de este orden, y muchos otros necesarios como los relativos a las diversas modalidades organizacionales existentes en participación. sus ventajas y limitaciones, pueden contribuir a crear un fondo de conocimientos al respecto, que fortalecerá la acción concreta.

b) Debe realizarse una tarea continuada de "aprendizaje" de las experiencias exitosas de la región. Hay un importante caudal de experiencias de este tipo como las numerosas presentadas en el pionero Encuentro del BID "Programas Sociales, pobreza y participación ciudadana" (1998). Es muy limitada la tarea de documentos de dichas experiencias, y revisión de sus enseñanzas. Hay en ese "rescate del conocimiento acumulado" una amplia línea de trabajo a seguir.

c) Se debe apoyar la realización de nuevas experiencias innovadoras en este campos. La participación significa una experimentación social compleja. Trabaja con variables multifacéticas culturales, ambientales, organizacionales, económicas, financieras, políticas, demográficas, etc. Es abierta para el desarrollo de innovaciones en todas sus etapas, que luego pueden ser aprovechadas colectivamen- te. Pero se requiere para ello como en otros campos, políticas de apoyo a la realización de experiencias innovativas.

Así por ejemplo entre otros casos en el Gobiemo del Canadá, el premio 1991 a la Administración innovativa en el área pública fue dedicado al tema: "Participación: empleados, gerentes, organizaciones". La existencia de un premio de esta índole motivó 68 representaciones de experiencias de todos los niveles del gobierno canadiense.

Las enseñanzas derivadas de las mismas han dado lugar a múltiples análisis, que a su vez están retroalimentando a otras experiencias y proyectos.

d) Es necesario forjar una gran alianza estratégica en torno de la participación. Diversos actores sociales tienen alto interés en su avance. Normalmente sus esfuerzos son aislados. Su articulación a niveles sectoriales y nacionales puede dar fuerza renovada a la acción: Entre ellos aparecen actores como los Municipios, las organizaciones no gubernamentales, Universidades, asociaciones vecinales, comunidades religiosas que trabajan en el campo social, diversos organismos internacionales, y desde ya las comunidades desfavorecidas.

El trabajo conjunto de estos y otros sectores para impulsar la participación, proteger experiencias en marcha, buscar comprometer sectores cada vez más amplios, obtener recursos en su apoyo, fortalecer la investigación y otros planos de acción, puede mejorar significativamente las condiciones para su aplicabilidad. 
e) Un punto central a encarar, que puede ser uno de los ejes de trabajo de la alianza estratégica, es la generación de conciencia pública respecto a las ventajas de la participación. Es necesario procurar que el tema trascienda la discusión de los especialistas, y se convierta en una cuestión de la agenda pública dadas sus implicancias de todo orden. Se requiere una tarea intensiva con medios masivos de comunicación sobre la materia. Asimismo nutrir la discusión con información detallada sobre todos los aspectos: potencial, dificultades esperables, experiencias internacionales, enseñanzas de las experiencias realizadas y en marcha. Dada la genuinidad de la pro puesta de la participación una opinión pública informada al respecto puede ser un activo factor en su favor.

\section{Sexta tesis. La participación se halla en la naturaleza misma del ser humano}

El informe sobre Desarrollo Humano de las Naciones Unidas centrado en participación (1993) señala:

"Una participación mayor de la población no es más una vaga ideología basada en los buenos deseos de unos pocos idealistas. Se ha convertido en un imperativo - una condición de supervivencia".

Este es claramente el caso de la participación en América Latina. Tanto en el campo general de fortalecimiento de los procesos de democratización, como en el de enfrentar los graves problemas sociales que afectan duramente a la mayor parte de la población.

Pero aún hay más. Como lo señalara Enrique V. Iglesias (1998), en sus palabras de cierre de la magna reunión convocada por el BID sobre el tema, la participación implica devolver a la población un derecho que le pertenece.

Efectivamente, en la identidad básica del ser humano, se halla la necesidad de la participación. Un profundo conocedor del tema, Juan XXIII, resaltó en su encíclica Mater et Magistra (1961) que el designio divino ha creado a los seres humanos de tal modo que: "en la naturaleza de los hombres se halla involucrada la exigencia de que en el desenvolvimiento de su actividad productora, tengan posibilidad de empeñar la propia responsabilidad y perfeccionar el propio ser". El involucramiento es una exigencia interna de la naturaleza misma del ser humano.

La participación comunitaria es un instrumento potente como se ha marcado en este trabajo, pero nunca debe perderse de vista que es el al mismo tiempo un fin en sí misma. Hace a la naturaleza del ser humano participar.

La participación eleva su dignidady le abre posibilidades de desarrollo y realización. Trabajar por la participación es en definitiva hacerlo por restituir a los desfavorecidos de América Latina uno de los derechos humanos más básicos, que con frecuencia - silenciosamente- les ha sido conculcado.

\section{Bibliografía citada}

Banco Interamericano de Desarrollo (BID 1997) Libro de consulta sobre participaclón. BID, 1997. 
BID, PNUD, CEPAL. Informe de la Comisión Latinoamericana y del Caribe sobre el Desarrollo Social (Comisión presidida por Patricio Aylwin), 1995.

Beaulieu, Rémy and Manoukian Violeta. Participatory Development: a brief review of CIDA's experience and potencial, Canadian Internacional Development Agency, 1994.

Blustein, Paul. "Missionary word" The Washington Post Magazine, November 10, 1996.

Burki, Shadid Javed. Opening Statement. En "Poverty \& Inequality". Annual Worid Bank Conference on Development in Latin America and the Caribbean. World Bank, 1996.

"Declaración de Santiago". Segunda Reunión de las Américas. Chile, 1998

Drucker, Peter. Conferencia en Drucker Foundation Advisory board, 1993. Citado por Goldschmidt, Marshall. "Ask, learn, follow up and grow. Hesselbein, Goldschmidt, Beckhard The leader of the future. Drucker Foundation, 1996.

Franco, Carios. "Imágenes de Villa El Salvador". En B. Kliksberg (comp.) ¿Cómo enfrentar la pobreza? Grupo Editor Latinoamericano, 1992.

Gai, Dharam (1989). "Desarrollo con participación: algunas perspectivas basadas en experiencia de origen popular" Revista de la planificación del desarrollo, No. 19.

Goldschmidt, Marshall, "Ask, learn, follow up and grow", en obra citada.

Hackman, J. R. "The psicology of self management in organizations". En pollak, M.S. and Perlof, R. Psycology and work. American Psycological Association. Washington, 1986.

Hirschman, Albert $O$. "Against parsimony: there easy ways of complicating some categories of economic discurse". American Economic Review, 1984

Iglesias Enrique V. palabras de cierre, Seminario Programas sociales, pobreza y participación ciudadana. BID, 1998.

Isham, Jonathan, Narayan, Deepa, and Pritchett, Lant. Does participation improve performarce? Establishing causalitty with subjective data. World Bank, 1995

Juan XXIII. Carta Enciclica Mater et Magistra. Librería Católica Acción. Buenos Alres, 1961.

Kernagham, Kenneth. "¿Facultamiento y Administración Pública: un avance revolucionario o una tendencia pasajera?" Canadian Public Administratión, Vol. 32, No. 2, 1994.

Kotter, John. "¿Qué hacen los gerentes realmente eficaces?" Harvard Business Review, November- December, 1998.

Leavitt, Harold J. and Jean Lipman- Blumen. "Hot Groups". Harvard Businness Review, July - August, 1995

Merton, Robert. Teoria y Estructura Sociales. Fondo de cultura Económica, 1964.

Mintzberg, Henry. "The manager's job: folklore and fact." En Kolb, Osland, Rubin. The Organizational Behavior Reader. Prentice Hall, 1995

Navarro, Zander "La democracia afirmativa y el desarrollo redistributivo: El caso del presupuesto participativo en Porto Alegre, Brasil (1989-1998)" Seminario Programas Sociales, Pobreza, y participación Ciudadana. BID; 1998

Narayan, Deepa. "The contribution of people's participation: 121 rural water supply proyects." World Bank, Workshop on participatory development, 1994.

Organization for Economic Cooperation and Development (OECD). Orientations 
on participatory development and good governance. Paris, 1993.

Peters, T.J. Driving on chaos. Knopt, New York, 1988.

Programa de las Naciones Unidas para el Desarrollo (PNUD 1993) Informe sobre desarrollo Humano.

Puryear, Jeffrey. La educación en América Latina: Problemas y desafios. Programas de Promoción de la Reforma Educativa en América Latina (PREAL), Washington, 1998.

Racelis, Mary. "Movilizando la población para el desarrollo social. Entoques y técnicas para la participación popular". Incluido en Bernardo, Kliksberg (comp.) Pobreza. Un tema impostergable. Nuevas respuestas a nivel mundial, $4^{a}$ edic, Fondo de Cultura Económica, 1997.

Schelp, Lothar. "The role of organizations in community participation. Prevention of accidental injuries in a rural Swedish municipality". Social Science and Medicine. United Kingdom, Vol.26. 1988.

Senge, Peter. La quinta disciplina. Granica, 1992

Sen, Amartya. On Ethics and Economics. Basil Blackwell Ltd., 1987

Stein, Alfredo. "Community participation in social proyects: the experience of the lo- cal development program (PRODEL) in Nicaragua". Seminario Programas Sociales, Pobreza, y Participación Cludadana. BID, 1998.

Tannembaum, A.S., y otros. Hierarchy in Organizations. Jossey Bass, 1974.

The New York Times. Growth 's limits in Latin America. May 6, 1997

The World Bank. Participation sourcebook, 1996.

The world Bank. Report on world development, 1996.

The World Bank, Operations Policy Departament. The Wordl Bank and Participation. 1994.

Walton, Aichard E. "From control to commitment in the workplace". En Kolb, Osland, Rubin, The organizational behavior. Prentice hall, 1995.

Wilpert, Bernhard. "Participation in organizations: evidence from international comparative research". International Science Journal, Vol. 36 , No. 2 , 1984.

Zapata, Gastón.(1992) una estrategia de desarrollo alternativo basada en la Participación Social y la Organización Comunitaria. Villa el Salvador. Incluido en B. Kliksberg ¿Cómo entrentar la pobreza? Grupo Editor Latinoamericano. 